\title{
A Kantorovich variant of a generalized Bernstein operators
}

\author{
Arun Kajla ${ }^{a}$, Praveen Agarwal $^{b}$, Serkan Aracic,* \\ ${ }^{a}$ Department of Mathematics, Central University of Haryana, Haryana-123031, India. \\ ${ }^{b}$ Department of Mathematics, Anand International College of Engineering, Jaipur, Rajasthan, India. \\ ${ }^{c}$ Department of Economics, Faculty of Economics, Administrative and Social Sciences, Hasan Kalyoncu University, TR-27410 \\ Gaziantep, Turkey.
}

\begin{abstract}
In this note we present a Kantorovich variant of the operators proposed by [X. Y. Chen, J. Q. Tan, Z. Liu, J. Xie, J. Math. Anal. Appl., 450 (2017), 244-261] based on non-negative parameters. Here, we prove an approximation theorem with the help of Bohman-Korovkin's principle and study the estimate of the rate of approximation by using the modulus of smoothness and Lipschitz type function for these operators. Also, we establish Voronovskaja type theorem and Korovkin type A-statistical approximation theorem of these operators.
\end{abstract}

Keywords: Global approximation, rate of convergence, modulus of continuity, A-statistical convergence, Kantorovich operators. 2010 MSC: 41A25, 26A15.

(C)2019 All rights reserved.

\section{Introduction}

For $f \in C(I)$, with $I=[0,1]$, the classical Bernstein polynomials are defined as follows:

$$
\mathcal{B}_{n}(f ; x)=\sum_{k=0}^{n} p_{n, k}(x) f\left(\frac{k}{n}\right), \quad x \in I,
$$

where $p_{n, k}(x)=\left(\begin{array}{l}n \\ k\end{array}\right) x^{k}(1-x)^{n-k}$ is the Bernstein basis.

Chen et al. [10] introduced a generalization of the Bernstein operators based on any fixed real $\alpha$ as follows:

$$
T_{n}^{(\alpha)}(f ; x)=\sum_{k=0}^{n} p_{n, k}^{(\alpha)}(x) f\left(\frac{k}{n}\right), \quad x \in I,
$$

\footnotetext{
*Corresponding author

Email addresses: rachitkajla47@gmail.com (Arun Kajla), goyal.praveen2011@gmail.com (Praveen Agarwal),

mtsrkn@hotmail.com (Serkan Araci)
}

doi: $10.22436 /$ jmcs.019.02.03

Received: 2017-11-21 Revised: 2018-05-11 Accepted: 2019-04-01 
where $p_{n, k}^{(\alpha)}(x)=\left[\left(\begin{array}{c}n-2 \\ k\end{array}\right)(1-\alpha) x+\left(\begin{array}{c}n-2 \\ k-2\end{array}\right)(1-\alpha)(1-x)+\left(\begin{array}{c}n \\ k\end{array}\right) \alpha x(1-x)\right] x^{k-1}(1-x)^{n-k-1}$ and $n \geqslant 2$. They proved the rate of convergence, Voronovskaja type asymptotic formula and shape preserving properties for these operators. In the special case $\alpha=1$ these operators reduce to the well-known Bernstein operators.

Razi [27] introduced a Bernstein-Kantorovich operator involving Pólya-Eggenberger distribution. He obtained the degree of approximation and the rate of convergence for these operators. Özarslan and Duman [26] considered a sequence of modified Bernstein-Kantorovich operators based on nonnegative parameter and studied the rate of convergence and global approximation of these operators. Abel and Heilmann [1] studied the complete asymptotic expansion of the Bernstein-Durrmeyer operators. Gonska and Paltanea [20] introduced genuine Bernstein-Durrmeyer operators involving one parameter family of linear positive operators and studied the simultaneous approximation for these operators. CárdenasMorales and Gupta [9] considered the two-parameter family of Bernstein-Durrmeyer type operators involving Polya distribution and obtained the approximation properties of these operators. Gupta et al. [21] proposed the Durrmeyer variant of the Baskakov operators based on inverse Pòlya-Eggenberger distribution and present the direct results. For Kantorovich-type generalizations of linear positive operators, we refer to some articles such as $[2-4,7,8,11,12,19,22]$, etc..

Inspired by their work, for $f \in C(I), a>0$, and $\rho>0$, we present the following Kantorovich type modification of the operators (1.1) as:

$$
\mathcal{K}_{n, \rho}^{\alpha, a}(f ; x)=\sum_{k=0}^{n} p_{n, k}^{(\alpha)}(x) \int_{0}^{1} f\left(\frac{k+a t^{\rho}}{n+a}\right) d t, \quad x \in I,
$$

where $p_{n, k}^{(\alpha)}(x)$ is as given in (1.1). For the special case $a=0$, these operators reduce to the operators (1.1).

In this article, we prove the basic approximation theorem for the operators (1.2) by using BohmanKorovkin's theorem. We also obtain estimates of the rate of convergence involving modulus of continuity and Lipschitz function. Also, we discuss A-statistical convergence for these operators using Korovkin type statistical convergence theorem.

Let $e_{i}(x)=x^{i}, i=0,1,2, \ldots$

Lemma 1.1. For the operators $\mathcal{K}_{n, \rho}^{\alpha, a}(f ; x)$, we have

(i) $\mathcal{K}_{n, \rho}^{\alpha, a}\left(e_{0} ; x\right)=1$;

(ii) $\mathcal{K}_{n, \rho}^{\alpha, a}\left(e_{1} ; x\right)=\frac{n x}{(n+a)}+\frac{a}{(n+a)(1+\rho)}$;

(iii) $\mathcal{K}_{n, \rho}^{\alpha, a}\left(e_{2} ; x\right)=\frac{x^{2}\left(n^{2}-n+2 \alpha-2\right)}{(n+a)^{2}}+\frac{x(n(\rho+2 a+1)-2(1+\rho)(\alpha-1))}{(n+a)^{2}(1+\rho)}+\frac{a^{2}}{(n+a)^{2}(1+2 \rho)}$;

(iv) $\mathcal{K}_{n, \rho}^{\alpha, a}\left(e_{3} ; x\right)=\frac{x^{3}(n-2)\left(n^{2}-n+6 \alpha-6\right)}{(n+a)^{3}}+\frac{3 x^{2}\left(a\left(n^{2}-n+2 \alpha-2\right)+(1+\rho)\left(n^{2}+n-2 n \alpha+6 \alpha-6\right)\right)}{(n+a)^{3}(1+\rho)}$

$+\frac{x\left(3 a^{2} n(1+\rho)+3 a(n-2 \alpha+2)(1+2 \rho)+(n-6 \alpha+6)\left(1+3 \rho+2 \rho^{2}\right)\right)}{(n+a)^{3}(1+\rho)(1+2 \rho)}+\frac{a^{3}}{(n+a)^{3}(1+3 \rho)} ;$

(v) $\mathcal{K}_{n, \rho}^{\alpha, a}\left(e_{4} ; x\right)=\frac{\chi^{4}(n-2)(n-3)(n(n-1)+12(\alpha-1))}{(n+a)^{4}}$

$+\frac{2 x^{3}(n-2)\left(2 a\left(n^{2}-n+6 \alpha-6\right)+3\left(n^{2}+n-2(n-6) \alpha-12\right)(1+\rho)\right)}{(n+a)^{4}(1+\rho)}$

$+\frac{x^{2}\left(6 a^{2}\left(n^{2}-n+2 \alpha-2\right)(1+\rho)+12 a\left(n^{2}+n-2 n \alpha+6 \alpha-6\right)(1+2 \rho)+(n(7 n-36 \alpha+29)+86(\alpha-1))(1+\rho)(1+2 \rho)\right)}{(n+a)^{4}(1+\rho)(1+2 \rho)}$

$+\frac{x\left(4 a^{3} n\left(2 \rho^{2}+3 \rho+1\right)+6 a^{2}(n-2 \alpha+2)\left(3 \rho^{2}+4 \rho+1\right)+4 a(n-6 \alpha+6)\left(6 \rho^{2}+5 \rho+1\right)+(n-14 \alpha+14)\left(6 \rho^{3}+11 \rho^{2}+6 \rho+1\right)\right)}{(n+a)^{4}(1+\rho)(1+2 \rho)(1+3 \rho)}$

$+\frac{a^{4}}{(n+a)^{4}(1+4 \rho)}$.

Let $e_{i}^{x}(t)=(t-x)^{i}, i=1,2$.

Lemma 1.2. For the operators $\mathcal{K}_{n, \rho}^{\alpha, a}(f ; x)$, we get

(i) $\mathcal{K}_{n, \rho}^{\alpha, a}\left(e_{1}^{\chi}(t) ; x\right)=\frac{-a x}{(n+a)}+\frac{a}{(n+a)(1+\rho)}$; 
(ii) $\mathcal{K}_{n, \rho}^{\alpha, a}\left(e_{2}^{\chi}(t) ; x\right)=\frac{x^{2}\left(a^{2}+2 \alpha-n-2\right)}{(n+a)^{2}}+\frac{x\left((1+\rho)(n-2 \alpha+2)-2 a^{2}\right)}{(n+a)^{2}(1+\rho)}+\frac{a^{2}}{(n+a)^{2}(1+2 \rho)}$.

Proof. Using Lemma 1.1, the proof of this lemma readily follows. Hence, the details are missing.

Remark 1.3. Let $\Theta_{n, \rho}^{\alpha, m, a}:=\mathcal{K}_{n, \rho}^{\alpha, a}\left((t-x)^{m} ; x\right), m=1,2,4$ be the central moments of $\mathcal{K}_{n, \rho}^{\alpha, a}$, we get

$$
\lim _{n \rightarrow \infty} n \Theta_{n, \rho}^{\alpha, 1, a}(x)=-a x+\frac{a}{(1+\rho)}, \quad \lim _{n \rightarrow \infty} n \Theta_{n, \rho}^{\alpha, 2, a}(x)=x(1-x), \quad \lim _{n \rightarrow \infty} n^{2} \Theta_{n, \rho}^{\alpha, 4, a}(x)=3 x^{2}(1-x)^{2} .
$$

Lemma 1.4. For $\mathrm{f} \in \mathrm{C}(\mathrm{I})$, we have

$$
\left\|\mathcal{K}_{n, \rho}^{\alpha, a}(f ; x)\right\| \leqslant\|f\| .
$$

Proof. Applying the definition (1.2) and Lemma 1.1, we get

$$
\left\|\mathcal{K}_{n, \rho}^{\alpha, a}(f ; x)\right\| \leqslant \sum_{k=0}^{n} p_{n, k}^{(\alpha)}(x) \int_{0}^{1}\left|f\left(\frac{k+a t^{\rho}}{n+a}\right) d t\right| d t \leqslant\|f\| \mathcal{K}_{n, \rho}^{\alpha, a}\left(e_{0} ; x\right)=\|f\| .
$$

Theorem 1.5. Let $\mathrm{f} \in \mathrm{C}(\mathrm{I})$. Then $\lim _{\mathrm{n} \rightarrow \infty} \mathcal{K}_{\mathrm{n}, \rho}^{\alpha, \mathrm{a}}(\mathrm{f} ; \mathrm{x})=\mathrm{f}(\mathrm{x})$, uniformly in $\mathrm{I}$.

Proof. Since $\mathcal{K}_{n, \rho}^{\alpha, a}(1 ; x)=1, \mathcal{K}_{n, \rho}^{\alpha, a}(t ; x) \rightarrow x, \mathcal{K}_{n, \rho}^{\alpha, a}\left(t^{2} ; x\right) \rightarrow x^{2}$ as $n \rightarrow \infty$, uniformly in I. By BohmanKorovkin's theorem, it follows that $\mathcal{K}_{\mathfrak{n}, \rho}^{\alpha, a}(f ; x)$ converges to $f(x)$ uniformly on $I$.

\section{Local approximation}

We begin by recalling the first and second order modulus of smoothness of $f \in C(I)$ are defined, respectively, by

$$
\omega(f, \delta)=\sup _{0<h \leqslant \delta} \sup _{x, x+h \in I}|f(x+h)-f(x)|
$$

and

$$
\omega_{2}(f, \sqrt{\delta})=\sup _{0<h \leqslant \sqrt{\delta}} \sup _{x, x+2 h \in I}|f(x+2 h)-2 f(x+h)+f(x)| .
$$

Let us consider the following K-functional

$$
K_{2}(f, \delta)=\inf \left\{\|f-g\|+\delta\left\|g^{\prime \prime}\right\|: g \in W^{2}\right\}(\delta>0),
$$

where $W^{2}=\left\{g: g^{\prime \prime} \in C(I)\right\}$ and $\|$.$\| is the uniform norm on C(I)$. In [13], there exists a positive constant $C>0$ such that

$$
K_{2}(f, \delta) \leqslant C \omega_{2}(f, \sqrt{\delta}) .
$$

Theorem 2.1. For the operators $\mathcal{K}_{\mathfrak{n}, \rho}^{\alpha, a}$, there exists a positive constant $\mathrm{C}>0$ such that

$$
\left|\mathcal{K}_{n, \rho}^{\alpha, a}(f ; x)-f(x)\right| \leqslant C \omega_{2}\left(f, \sqrt{\mu_{n, \rho}^{\alpha, a}(x)}\right)+w\left(f,(n+a)^{-1}\right),
$$

where $\mu_{n, \rho}^{\alpha, a}(x)=\frac{x^{2}\left(2 a^{2}+2 \alpha-n-2\right)}{(n+a)^{2}}+\frac{x\left((1+\rho)(n+2-2 \alpha)-4 a^{2}\right)}{(n+a)^{2}(1+\rho)}+\frac{a^{2}(2+\rho(4+\rho))}{(n+a)^{2}(1+\rho)^{2}(1+2 \rho)}, f \in C(I)$, and $x \in I$.

Proof. Let

$$
\mathcal{T}_{n, \rho}^{\alpha, a}(f ; x)=\mathcal{K}_{n, \rho}^{\alpha, a}(f ; x)+f(x)-f\left(\frac{n x}{(n+a)}+\frac{a}{(n+a)(1+\rho)}\right)
$$


Then, in view of Lemma 1.1, we have

and

$$
\mathcal{T}_{n, \rho}^{\alpha, a}(1 ; x)=\mathcal{K}_{n, \rho}^{\alpha, a}(1 ; x)=1
$$

$$
\mathcal{T}_{n, \rho}^{\alpha, a}(t ; x)=\mathcal{K}_{n, \rho}^{\alpha, a}(t ; x)+x-\left(\frac{n x}{(n+a)}+\frac{a}{(n+a)(1+\rho)}\right)=x
$$

Let $g \in W^{2}$ and $t \in I$. By Taylor's formula we find

$$
g(t)=g(x)+(t-x) g^{\prime}(x)+\int_{x}^{t}(t-u) g^{\prime \prime}(u) d u .
$$

Operating the operator $\mathcal{T}_{n, \rho}^{\alpha, a}$ on both sides of the above equation, we get

$$
\begin{aligned}
\mathcal{T}_{n, \rho}^{\alpha, a}(g ; x)= & g(x)+\mathcal{T}_{n, \rho}^{\alpha, a}\left(\int_{x}^{t}(t-u) g^{\prime \prime}(u) d u ; x\right) \\
= & g(x)+\mathcal{K}_{n, \rho}^{\alpha, a}\left(\int_{x}^{t}(t-u) g^{\prime \prime}(u) d u ; x\right) \\
& -\int_{x}^{\frac{n x}{(n+a)}+\frac{a}{(n+a)(1+\rho)}}\left(\frac{n x}{(n+a)}+\frac{a}{(n+a)(1+\rho)}-u\right) g^{\prime \prime}(u) d u .
\end{aligned}
$$

Therefore,

$$
\begin{aligned}
\left|\mathcal{T}_{n, \rho}^{\alpha, a}(g ; x)-g(x)\right| \leqslant & \mathcal{K}_{n, \rho}^{\alpha, a}\left(\left|\int_{x}^{t}\right| t-u \| g^{\prime \prime}(u)|d u| ; x\right) \\
& +\left|\int_{x}^{\frac{n x}{(n+a)}+\frac{a}{(n+a)(1+\rho)}}\right| \frac{n x}{(n+a)}+\frac{a}{(n+a)(1+\rho)}-u|| g^{\prime \prime}(u)|d u| \\
\leqslant & \left\{\mathcal{K}_{n, \rho}^{\alpha, a}\left((t-x)^{2} ; x\right)+\left(\frac{n x}{(n+a)}+\frac{a}{(n+a)(1+\rho)}-x\right)^{2}\right\}\left\|g^{\prime \prime}\right\| \\
= & \left\{\frac{x^{2}\left(2 a^{2}+2 \alpha-n-2\right)}{(n+a)^{2}}+\frac{x\left((1+\rho)(n+2-2 \alpha)-4 a^{2}\right)}{(n+a)^{2}(1+\rho)}\right. \\
& \left.+\frac{a^{2}(2+\rho(4+\rho))}{(n+a)^{2}(1+\rho)^{2}(1+2 \rho)}\right\}\left\|g^{\prime \prime}\right\| \\
= & \mu_{n, \rho}^{\alpha, a}(x)\left\|g^{\prime \prime}\right\| .
\end{aligned}
$$

Using Lemma 1.4, we get

$$
\left|\mathcal{T}_{n, \rho}^{\alpha, a}(f ; x)\right| \leqslant\left|\mathcal{K}_{n, \rho}^{\alpha, a}(f ; x)\right|+|f(x)|+\left|f\left(\frac{n x}{(n+a)}+\frac{a}{(n+a)(1+\rho)}\right)\right| \leqslant 3\|f\|, \quad \forall f \in C(I) .
$$

Now, for $g \in W^{2}$ and $f \in C(I)$, using (2.3) and (2.4), we get

$$
\begin{aligned}
\left|\mathcal{K}_{n, \rho}^{\alpha, a}(f ; x)-f(x)\right| \leqslant & \left|\mathcal{T}_{n, \rho}^{\alpha, a}(f ; x)-f(x)+f\left(\frac{n x}{(n+a)}+\frac{a}{(n+a)(1+\rho)}\right)-f(x)\right| \\
\leqslant & \left|\mathcal{T}_{n, \rho}^{\alpha, a}(f-g ; x)\right|+\left|\mathcal{T}_{n, \rho}^{\alpha, a}(g ; x)-g(x)\right|+|g(x)-f(x)| \\
& +\left|f\left(\frac{n x}{(n+a)}+\frac{a}{(n+a)(1+\rho)}\right)-f(x)\right| \\
\leqslant & 4\|f-g\|+\mu_{n, \rho}^{\alpha, a}(x)\left\|g^{\prime \prime}\right\|+\omega\left(f,(n+a)^{-1}\right) .
\end{aligned}
$$


Taking the infimum on the right hand side over all $g \in W^{2}$, we have

$$
\left|\mathcal{K}_{n, \rho}^{\alpha, a}(f ; x)-f(x)\right| \leqslant 4 K_{2}\left(f, \mu_{n, \rho}^{\alpha, a}(x)\right)+\omega\left(f,(n+a)^{-1}\right),
$$

and using the relation (2.1), we get

$$
\left|\mathcal{K}_{n, \rho}^{\alpha, a}(f ; x)-f(x)\right| \leqslant M \omega_{2}\left(f, \sqrt{\mu_{n, \rho}^{\alpha, a}(x)}\right)+w\left(f,(n+a)^{-1}\right),
$$

hence the theorem is proved .

Let us consider the Lipschitz-type space with two parameters [25]. For $\beta_{1} \geqslant 0, \beta_{2}>0$, we define

$$
\operatorname{Lip}_{M}^{\left(\beta_{1}, \beta_{2}\right)}(\eta):=\left\{f \in C(I):|f(t)-f(x)| \leqslant M \frac{|t-x|^{\eta}}{\left(t+\beta_{1} x^{2}+\beta_{2} x\right)^{\frac{\eta}{2}}} ; t \in I, x \in(0,1]\right\},
$$

where $0<\eta \leqslant 1$.

Theorem 2.2. Let $\mathrm{f} \in \operatorname{Lip}_{M}^{\left(\beta_{1}, \beta_{2}\right)}(\eta)$. Then for all $x \in(0,1]$, we have

$$
\left|\mathcal{K}_{n, \rho}^{\alpha, a}(f ; x)-f(x)\right| \leqslant M\left(\frac{\Theta_{n, \rho}^{\alpha, 2, a}(x)}{\beta_{1} x^{2}+\beta_{2} x}\right)^{\eta / 2},
$$

where $\Theta_{n, \rho}^{\alpha, 2, a}(x)=\mathcal{K}_{n, \rho}^{\alpha, a}\left((t-x)^{2} ; x\right)$.

Proof. Let we prove the theorem for the case $0<\eta \leqslant 1$, applying Holder's inequality with $p=\frac{2}{\eta}, q=\frac{2}{2-\eta}$,

$$
\begin{aligned}
\left|\mathcal{K}_{n, \rho}^{\alpha, a}(f ; x)-f(x)\right| & \leqslant \sum_{k=0}^{n} p_{n, k}^{(\alpha)}(x) \int_{0}^{1}\left|f\left(\frac{k+a t^{\rho}}{n+a}\right)-f(x)\right| d t \\
& \leqslant \sum_{k=0}^{n} p_{n, k}^{(\alpha)}(x)\left(\int_{0}^{1}\left|f\left(\frac{k+a t^{\rho}}{n+a}\right)-f(x)\right|^{\frac{2}{\eta}} d t\right)^{\frac{\eta}{2}} \\
& \leqslant\left\{\sum_{k=0}^{n} p_{n, k}^{(\alpha)}(x) \int_{0}^{1}\left|f\left(\frac{k+a t^{\rho}}{n+a}\right)-f(x)\right|^{\frac{2}{\eta}} d t\right\}^{\frac{\eta}{2}}\left(\sum_{k=0}^{n} p_{n, k}^{(\alpha)}(x)\right)^{\frac{2-n}{2}} \\
& =\left(\sum_{k=0}^{n} p_{n, k}^{(\alpha)}(x) \int_{0}^{1}\left|f\left(\frac{k+a t^{\rho}}{n+a}\right)-f(x)\right|^{\frac{2}{\eta}} d t\right)^{\frac{\eta}{2}} \\
& \leqslant M\left(\sum_{k=0}^{n} p_{n, k}^{(\alpha)}(x) \int_{0}^{1} \frac{\left(\frac{k+a t^{\rho}}{n+a}-x\right)^{2}}{\left(\frac{k+a t^{\rho}}{n+a}+\beta_{1} x^{2}+\beta_{2} x\right)} d t\right)^{\frac{\eta}{2}} \\
& \leqslant \frac{M}{\left(\beta_{1} x^{2}+\beta_{2} x\right)^{\frac{\eta}{2}}}\left(\sum_{k=0}^{n} p_{n, k}^{(\alpha)}(x) \int_{0}^{1}\left(\frac{k+a t^{\rho}}{n+a}-x\right)^{2} d t\right)^{\frac{\eta}{2}} \\
& =\frac{M}{\left(\beta_{1} x^{2}+\beta_{2} x\right)^{\frac{n}{2}}} \mathcal{K}_{n, \rho}^{\alpha, a}\left((t-x)^{2} ; x\right)^{\frac{\eta}{2}}=\frac{M}{\left(\beta_{1} x^{2}+\beta_{2} x\right)^{\frac{n}{2}}}\left(\Theta_{n, \rho}^{\alpha, 2, a}(x)\right)^{\frac{\eta}{2}}
\end{aligned}
$$

Theorem 2.3. Let $\mathrm{f} \in \mathrm{C}(\mathrm{I})$. Then,

$$
\left|\mathcal{K}_{n, \rho}^{\alpha, a}(f ; x)-f(x)\right| \leqslant 2 \omega\left(f ; \sqrt{\Theta_{n, \rho}^{\alpha, 2, a}(x)}\right) .
$$


Proof. Using the following well-known property of modulus of continuity,

$$
|f(t)-f(x)| \leqslant \omega(f, \delta)\left(\frac{(t-x)^{2}}{\delta^{2}}+1\right), \quad \text { for any } \delta>0,
$$

we get

$$
\left|\mathcal{K}_{n, \rho}^{\alpha, a}(f ; x)-f(x)\right| \leqslant \mathcal{K}_{n, \rho}^{\alpha, a}(|f(t)-f(x)| ; x) \leqslant\left(1+\frac{1}{\delta^{2}} \mathcal{K}_{n, \rho}^{\alpha, a}\left((t-x)^{2} ; x\right)\right) \omega(f, \delta) .
$$

Applying Lemma 1.2, it follows that $\left.\lim _{n \rightarrow \infty} \mathcal{K}_{n, \rho}^{\alpha, a}\left((t-x)^{2}\right) ; x\right)=0$, hence choosing $\delta=\sqrt{\Theta_{n, \rho}^{\alpha, 2, a}(x)}$, we obtain the result.

Theorem 2.4. For any $\mathrm{f} \in \mathrm{C}^{1}(\mathrm{I})$ and $\mathrm{x} \in[0,1]$, we have

$$
\left|\mathcal{K}_{n, \rho}^{\alpha, a}(f ; x)-f(x)\right| \leqslant\left|\frac{-a x}{(n+a)}+\frac{a}{(n+a)(1+\rho)}\right| f^{\prime}(x) \mid+2 \sqrt{\Theta_{n, \rho}^{\alpha, 2, a}(x)} \omega\left(f^{\prime}, \sqrt{\Theta_{n, \rho}^{\alpha, 2, a}(x)}\right) .
$$

Proof. Let $f \in \mathrm{C}^{1}(\mathrm{I})$. For any $\mathrm{t} \in[0,1], x \in[0,1]$, we have

$$
f(t)-f(x)=f^{\prime}(x)(t-x)+\int_{x}^{t}\left(f^{\prime}(u)-f^{\prime}(x)\right) d u .
$$

Using $\mathcal{K}_{n, \rho}^{\alpha, a}(\cdot ; x)$ on both sides of the above equation, we have

$$
\mathcal{K}_{n, \rho}^{\alpha, a}\left(f(t)-f(x) ; q_{n}, x\right)=f^{\prime}(x) \mathcal{K}_{n, \rho}^{\alpha, a}(t-x ; x)+\mathcal{K}_{n, \rho}^{\alpha, a}\left(\int_{x}^{t}\left(f^{\prime}(u)-f^{\prime}(x)\right) d u ; x\right) .
$$

Using the well-known property of modulus of continuity $|f(t)-f(x)| \leqslant \omega(f, \delta)\left(\frac{|t-x|}{\delta}+1\right), \delta>0$, we obtain

$$
\left|\int_{x}^{t}\left(f^{\prime}(u)-f^{\prime}(x)\right) d u\right| \leqslant w\left(f^{\prime}, \delta\right)\left(\frac{(t-x)^{2}}{\delta}+|t-x|\right)
$$

it follows that

$$
\left|\mathcal{K}_{n, \rho}^{\alpha, a}(f ; x)-f(x)\right| \leqslant\left|f^{\prime}(x)\right| \quad\left|\mathcal{K}_{n, \rho}^{\alpha, a}(t-x ; x)\right|+\omega\left(f^{\prime}, \delta\right)\left\{\frac{1}{\delta} \mathcal{K}_{n, \rho}^{\alpha, a}\left((t-x)^{2} ; x\right)+\mathcal{K}_{n, \rho}^{\alpha, a}(|t-x| ; x)\right\} .
$$

From Cauchy-Schwarz inequality, we have

$$
\left|\mathcal{K}_{n, \rho}^{\alpha, a}(f ; x)-f(x)\right| \leqslant\left|f^{\prime}(x)\right|\left|\mathcal{K}_{n, \rho}^{\alpha, a}(t-x ; x)\right|+w\left(f^{\prime}, \delta\right)\left\{\frac{1}{\delta} \sqrt{\mathcal{K}_{n, \rho}^{\alpha, a}\left((t-x)^{2} ; x\right)}+1\right\} \sqrt{\mathcal{K}_{n, \rho}^{\alpha, a}\left((t-x)^{2} ; x\right)}
$$

Now, taking $\delta=\sqrt{\Theta_{n, \rho}^{\alpha, 2, a}(x)}$, we obtain (2.5).

\section{Global approximation}

For $f \in C(I)$, the first and second order Ditzian-Totik moduli of smoothness are defined by

$$
\omega_{\psi}(f, \delta)=\sup _{0<|h| \leqslant \delta, x+h \psi(x) \in I} \sup _{0}|f(x+\psi(x) h)-f(x)|
$$

and

$$
\omega_{2, \phi}(f, \sqrt{\delta})=\sup _{0<|h| \leqslant \sqrt{\delta}} \sup _{x, x \pm h \phi(x) \in I}|f(x+h \phi(x))-2 f(x)+f(x-h \phi(x))|,
$$


respectively and the corresponding K-functional is

$$
K_{2, \phi}(f, \delta)=\inf \left\{\|f-g\|+\delta\left\|\phi^{2} g^{\prime \prime}\right\|: g \in W^{2}(\phi)\right\}, \quad \delta>0,
$$

where $W^{2}(\phi)=\left\{g \in C(I): g^{\prime} \in A C[0,1], \phi^{2} g^{\prime \prime} \in C(I)\right\}$ and $g^{\prime} \in A C[0,1]$ means that $g^{\prime}$ is absolutely continuous on I. It is well known (see [14]) $K_{2, \phi}(f, \delta) \sim \omega_{2, \phi}(f, \sqrt{\delta})$ which means that there exists an absolute constant $\mathrm{C}>0$ such that

$$
\mathrm{C}^{-1} \omega_{2, \phi}(f, \sqrt{\delta}) \leqslant K_{2, \phi}(f, \delta) \leqslant \mathrm{C} \omega_{2, \phi}(f, \sqrt{\delta}),
$$

where $\phi$ is an admissible step-weight function on I.

Theorem 3.1. Let $\mathrm{f} \in \mathrm{C}(\mathrm{I})$ and $\mathrm{x} \in(0,1)$. Then, there is an absolute constant $\mathrm{C}>0$ such that

$$
\left|\mathcal{K}_{n, \rho}^{\alpha, a}(f ; x)-f(x)\right| \leqslant C \omega_{2, \phi}\left(f, \frac{\sqrt{\mu_{n, \rho}^{\alpha, a}(x)}}{\phi(x)}\right)+\omega_{\psi_{\rho, a}}\left(f,(n+a)^{-1}\right),
$$

where $\psi_{\rho, \mathrm{a}}(x)=\mathrm{a}(x(1+\rho)+1)$ and $\phi^{2}(x)=x(1-x)$.

Proof. Operating the operators $\mathcal{T}_{n, \rho}^{\alpha, a}$ defined in (2.2) to Taylor's expansion in a similar manner with the proof of Theorem 2.1, we get

$$
\begin{aligned}
\left|\mathcal{T}_{n, \rho}^{\alpha, a}(g ; x)-g(x)\right| \leqslant & \mathcal{K}_{n, \rho}^{\alpha, a}\left(\left|\int_{x}^{t}\right| t-u|| g^{\prime \prime}(u)|d u| ; x\right) \\
& +\left|\int_{x}^{\frac{n x}{(n+a)}+\frac{a}{(n+a)(1+\rho)}}\right| \frac{n x}{(n+a)}+\frac{a}{(n+a)(1+\rho)}-u|| g^{\prime \prime}(u)|d u| \\
\leqslant & \left\|\phi^{2} g^{\prime \prime}\right\| \mathcal{K}_{n, \rho}^{\alpha, a}\left(\left|\int_{x}^{t} \frac{|t-u|}{\phi^{2}(u)} d u\right| ; x\right) \\
& +\left\|\phi^{2} g^{\prime \prime}\right\|\left|\int_{x}^{\frac{n x}{(n+a)}+\frac{a}{(n+a)(1+\rho)}} \frac{\left|\frac{n x}{(n+a)}+\frac{a}{(n+a)(1+\rho)}-u\right|}{\phi^{2}(u)} d u\right| .
\end{aligned}
$$

Let $u=\beta x+(1-\beta) t, \beta \in[0,1]$. Since $\phi^{2}(x)$ is concave on $I$, it follows that $\phi^{2}(u) \geqslant \beta \phi^{2}(x)+(1-\beta) \phi^{2}(t)$ and hence

$$
\frac{|t-u|}{\phi^{2}(u)}=\frac{\beta|x-t|}{\phi^{2}(u)} \leqslant \frac{\beta|x-t|}{\beta \phi^{2}(x)+(1-\beta) \phi^{2}(t)} \leqslant \frac{|x-t|}{\phi^{2}(x)} .
$$

Thus,

$$
\left|\mathcal{T}_{n, \rho}^{\alpha, a}(g ; x)-g(x)\right| \leqslant \frac{\left\|\phi^{2} g^{\prime \prime}\right\|}{\phi^{2}(x)}\left[\mathcal{K}_{n, \rho}^{\alpha, a}\left((t-x)^{2} ; x\right)+\left(\frac{n x}{(n+a)}+\frac{a}{(n+a)(1+\rho)}-x\right)^{2}\right] \leqslant \frac{\left\|\phi^{2} g^{\prime \prime}\right\|}{\phi^{2}(x)} \mu_{n, \rho}^{\alpha, a}(x) .
$$

Now,

$$
\begin{aligned}
\left|\mathcal{K}_{n, \rho}^{\alpha, a}(f ; x)-f(x)\right| \leqslant & \left|\mathcal{T}_{n, \rho}^{\alpha, a}(f-g ; x)\right|+\left|\mathcal{T}_{n, \rho}^{\alpha, a}(g ; x)-g(x)\right|+|f(x)-g(x)| \\
& +\left|f\left(\frac{n x}{(n+a)}+\frac{a}{(n+a)(1+\rho)}\right)-f(x)\right| \\
\leqslant & 4|| f-g \|+\frac{\left\|\phi^{2} g^{\prime \prime}\right\|}{\phi^{2}(x)} \mu_{n, \rho}^{\alpha, a}(x)+\left|f\left(\frac{n x}{(n+a)}+\frac{a}{(n+a)(1+\rho)}\right)-f(x)\right| .
\end{aligned}
$$

Taking infimum on the right hand side of the above inequality over all $g \in W^{2}(\phi)$, we get

$$
\left|\mathcal{K}_{n, \rho}^{\alpha, a}(f ; x)-f(x)\right| \leqslant 4 K_{2, \phi}\left(f, \frac{\mu_{n, \rho}^{\alpha, a}(x)}{4 \phi^{2}(x)}\right)+\left|f\left(\frac{n x}{(n+a)}+\frac{a}{(n+a)(1+\rho)}\right)-f(x)\right| .
$$


Also,

$$
\begin{aligned}
\left|f\left(\frac{n x}{(n+a)}+\frac{a}{(n+a)(1+\rho)}\right)-f(x)\right| & =\left|f\left(x+\psi_{\rho, a}(x) \frac{\mathcal{K}_{n, \rho}^{\alpha, a}((t-x) ; x)}{\psi_{\rho, a}(x)}\right)-f(x)\right| \\
& \leqslant \omega_{\psi_{\rho, a}}\left(f, \frac{1}{\psi_{\rho, a}(x)}\left|\mathcal{K}_{n, \rho}^{\alpha, a}((t-x) ; x)\right|\right) \leqslant \omega_{\psi_{\rho, a}}\left(f,(n+a)^{-1}\right) .
\end{aligned}
$$

Thus,

$$
\left|\mathcal{K}_{n, \rho}^{\alpha, a}(f ; x)-f(x)\right| \leqslant 4 K_{2, \phi}\left(f, \frac{\mu_{n, \rho}^{\alpha, a}(x)}{4 \phi^{2}(x)}\right)+\omega_{\psi_{\rho, a}}\left(f,(n+a)^{-1}\right) .
$$

Now, applying (3.1), we obtain the required result.

\section{Voronovskaja type theorem}

In this section we establish Voronvoskaja type result for the $\mathcal{K}_{n, \rho}^{\alpha, a}$ operators.

Theorem 4.1. Let $\mathrm{f} \in \mathrm{C}(\mathrm{I})$. If $\mathrm{f}^{\prime \prime}$ exists at a point $\mathrm{x} \in \mathrm{I}$, then we have

$$
\lim _{n \rightarrow \infty} n\left[\mathcal{K}_{n, \rho}^{\alpha, a}(f ; x)-f(x)\right]=\left(-a x+\frac{a}{(1+\rho)}\right) f^{\prime}(x)+\frac{1}{2} x(1-x) f^{\prime \prime}(x) .
$$

Proof. Applying Taylor's expansion, we can write

$$
f(t)=f(x)+f^{\prime}(x)(t-x)+\frac{1}{2} f^{\prime \prime}(x)(t-x)^{2}+\varepsilon(t, x)(t-x)^{2},
$$

where $\lim _{t \rightarrow x} \varepsilon(t, x)=0$. By using the linearity of the operator $\mathcal{K}_{n, \rho}^{\alpha, a}$, we get

$$
\mathcal{K}_{n, \rho}^{\alpha, a}(f ; x)-f(x)=\mathcal{K}_{n, \rho}^{\alpha, a}((t-x) ; x) f^{\prime}(x)+\frac{1}{2} \mathcal{K}_{n, \rho}^{\alpha, a}\left((t-x)^{2} ; x\right) f^{\prime \prime}(x)+\mathcal{K}_{n, \rho}^{\alpha, a}\left(\varepsilon(t, x)(t-x)^{2} ; x\right) .
$$

Applying the Cauchy-Schwarz inequality, we obtain

$$
n \mathcal{K}_{n, \rho}^{\alpha, a}\left(\varepsilon(t, x)(t-x)^{2} ; x\right) \leqslant \sqrt{\mathcal{K}_{n, \rho}^{\alpha, a}\left(\varepsilon^{2}(t, x) ; x\right)} \sqrt{n^{2} \mathcal{K}_{n, \rho}^{\alpha, a}\left((t-x)^{4} ; x\right)} .
$$

In view of Theorem 1.5, we get

$$
\lim _{n \rightarrow \infty} \mathcal{K}_{n, \rho}^{\alpha, a}\left(\varepsilon^{2}(t, x) ; x\right)=\varepsilon^{2}(x, x)=0 .
$$

Combining (4.1)-(4.2) and Remark 1.3, we have

$$
\lim _{n \rightarrow \infty} n \mathcal{K}_{n, \rho}^{\alpha, a}\left(\varepsilon(t, x)(t-x)^{2} ; x\right)=0 .
$$

Hence

$$
\lim _{n \rightarrow \infty} n\left[\mathcal{K}_{n, \rho}^{\alpha, a}(f ; x)-f(x)\right]=\left(-a x+\frac{a}{(1+\rho)}\right) f^{\prime}(x)+\frac{1}{2} x(1-x) f^{\prime \prime}(x) .
$$

Thus the theorem follows immediately by using Remark 1.3.

\section{A-statistical approximation of Korovkin type}

Let $A=\left(a_{n k}\right)$ be a non-negative infinite summability matrix. For a given sequence $x:=(x)_{n}$, the A-transform of $x$ denoted by $A x:(A x)_{n}$ is defined as

$$
(A x)_{n}=\sum_{k=1}^{\infty} a_{n k} x_{k}
$$

provided the series converges for each $n$. A is called to be regular if $\lim _{n}(A x)_{n}=L$ whenever $\lim _{n}(x)_{n}=L$. 
Then $x=(x)_{n}$ is said to be A-statistically convergent to L, i.e., $s t_{A}-\lim _{n}(x)_{n}=L$ if for every $\epsilon>$ $0, \lim _{n:\left|x_{k}-L\right| \geqslant e} \sum_{n k}=0$. If we replace $A$ by $C_{1}$ then $A$ is a Cesaro matrix of order one and $A$-statistical convergence is reduced to the statistical convergence. Similarly, if $A=I$, the identity matrix then $A$ statistical convergence is called ordinary convergence. Many researchers have examined the statistical convergence properties for several sequences and classes of positive linear operators (cf. $[5,6,15-17,23$, 24]).

Gadjiev and Orhan [18] proved the following Bohman-Korovkin type approximation theorem for statistical convergence:

Theorem 5.1 ([18]). If the sequence of positive linear operators $\mathcal{P}_{\mathrm{n}}: \mathrm{C}[\mathrm{c}, \mathrm{d}] \rightarrow \mathrm{C}[\mathrm{c}, \mathrm{d}]$ satisfy the conditions st $-\lim _{n}\left\|\mathcal{P}_{n}\left(e_{i}\right)-e_{i}\right\|_{C[c, d]}=0$ with $e_{i}(t)=t^{i}, i=0,1,2$, then for any function $f \in C[c, d]$, we have

$$
s t-\lim _{n}\left\|\mathcal{P}_{n}(f)-f\right\|_{C[c, d]}=0 .
$$

It is to be observe that this result works well for a A-statistical convergence also.

Theorem 5.2. Let $\left(\mathrm{a}_{\mathrm{nk}}\right)$ be a non-negative regular summability matrix. For any $\mathrm{f} \in \mathrm{C}(\mathrm{I})$, we have

$$
s t_{A}-\lim _{n}\left\|\mathcal{K}_{n, \rho}^{\alpha, a}(f)-f\right\|_{C(I)}=0 .
$$

Proof. Let us consider $e_{i}(x)=x^{i}, i=0,1$, 2. It is sufficient to show that $s t_{A}-\lim _{n}\left\|\mathcal{K}_{n, \rho}^{\alpha, a}\left(e_{i} ; \cdot\right)-e_{i}\right\|_{C(I)}=0$, for $i=0,1,2$. It is clear that

$$
s t_{A}-\lim _{n}\left\|\mathcal{K}_{n, \rho}^{\alpha, a}\left(e_{0} ; \cdot\right)-e_{0}\right\|_{C(I)}=0
$$

From Lemma 1.1, we have

$$
s t_{A}-\lim _{n}\left\|\mathcal{K}_{n, \rho}^{\alpha, a}\left(e_{1} ; \cdot\right)-e_{1}\right\|_{C(I)}=\left|\frac{n x}{(n+a)}+\frac{a}{(n+a)(1+\rho)}-x\right| \leqslant\left|\frac{-a}{(n+a)}\right|+\frac{a}{(n+a)(1+\rho)} .
$$

For $\epsilon>0$, let us define the following sets:

$$
\begin{aligned}
E & :=\left\{n \in \mathbb{N}:\left\|\mathcal{K}_{n, \rho}^{\alpha, a}\left(e_{1} ; \cdot\right)-e_{1}\right\| \geqslant \epsilon\right\}, \\
E_{1} & :=\left\{n \in \mathbb{N}:\left|\frac{-a}{(n+a)}\right| \geqslant \frac{\epsilon}{2}\right\}, \\
E_{2} & :=\left\{n \in \mathbb{N}: \frac{a}{(n+a)(1+\rho)} \geqslant \frac{\epsilon}{2}\right\}
\end{aligned}
$$

Then, we obtain $E \subseteq E_{1}$ which implies that $\sum_{k \in E} a_{n k} \leqslant \sum_{k \in E_{1}} a_{n k}+\sum_{k \in E_{2}} a_{n k}$ and hence

$$
s t_{A}-\lim _{n}\left\|\mathcal{K}_{n, \rho}^{\alpha, a}\left(e_{1} ; \cdot\right)-e_{1}\right\|_{C(I)}=0 .
$$

In the similar manner it can be shown

$$
\begin{aligned}
s t_{A}-\lim _{n} \| & \mathcal{K}_{n, \rho}^{\alpha, a}\left(e_{2} ; \cdot\right)-e_{2} \|_{C(I)} \\
& \leqslant\left|\frac{x^{2}\left(n^{2}-n+2 \alpha-2\right)}{(n+a)^{2}}+\frac{x(n(\rho+2 a+1)-2(1+\rho)(\alpha-1))}{(n+a)^{2}(1+\rho)}+\frac{a^{2}}{(n+a)^{2}(1+2 \rho)}-x^{2}\right| \\
& \leqslant\left|\frac{a^{2}+n+2 a n-2 \alpha+2}{(n+a)^{2}}\right|+\left|\frac{(n(\rho+2 a+1)-2(1+\rho)(\alpha-1))}{(n+a)^{2}(1+\rho)}\right|+\frac{a^{2}}{(n+a)^{2}(1+2 \rho)} .
\end{aligned}
$$


For $\epsilon>0$, let us define the following sets:

$$
\begin{aligned}
F & :=\left\{n \in \mathbb{N}:|| \mathcal{K}_{n, \rho}^{\alpha, a}\left(e_{2} ; \cdot\right)-e_{2} \| \geqslant \epsilon\right\}, \\
F_{1} & :=\left\{n \in \mathbb{N}:\left|\frac{a^{2}+n+2 a n-2 \alpha+2}{(n+a)^{2}}\right| \geqslant \frac{\epsilon}{3}\right\}, \\
F_{2} & :=\left\{n \in \mathbb{N}:\left|\frac{(n(\rho+2 a+1)-2(1+\rho)(\alpha-1))}{(n+a)^{2}(1+\rho)}\right| \geqslant \frac{\epsilon}{3}\right\}, \\
F_{3} & :=\left\{n \in \mathbb{N}: \frac{a^{2}}{(n+a)^{2}(1+2 \rho)} \geqslant \frac{\epsilon}{3}\right\} .
\end{aligned}
$$

Then, we obtain $F \subseteq F_{1} \cup F_{2}$ which implies that $\sum_{k \in F} a_{n k} \leqslant \sum_{k \in F_{1}} a_{n k}+\sum_{k \in F_{2}} a_{n k}+\sum_{k \in F_{3}} a_{n k}$ and hence

$$
s t_{A}-\lim _{n}\left\|\mathcal{K}_{n, \rho}^{\alpha, a}\left(e_{2} ; \cdot\right)-e_{2}\right\|_{C(I)}=0 .
$$

This completes the proof of the theorem.

\section{Conclusion}

Motivated by Chen et al. [10], we gave a Kantorovich variant of the operators based on non-negative parameters. From here, we proved an approximation theorem with the help of Bohman-Korovkin's principle and studied the estimate of the rate of approximation by making use of the modulus of smoothness and Lipschitz type function for these operators. Finally we established Voronovskaja type theorem and Korovkin type A-statistical approximation theorem of these operators.

\section{Acknowledgment}

Third author of this paper is supported by the Research fund of Hasan Kalyoncu University in 2019.

\section{References}

[1] U. Abel, M. Heilmann, The complete asymptotic expansion for Bernstein-Durrmeyer operators with Jacobi weights, Mediterr. J. Math., 1 (2004), 487-499. 1

[2] A. M. Acu, Stancu-Schurer-Kantorovich operators based on q-integers, Appl. Math. Comput., 259 (2015), 896-907. 1

[3] P. N. Agrawal, M. Goyal, A. Kajla, q-Bernstein-Schurer-Kantorovich type operators, Boll. Unione Mat. Ital., 8 (2015), 169-180.

[4] F. Altomare, M. M. Cappelletti, V. Leonessa, On a generalization of Szasz-Mirakjan-Kantorovich operators, Results Math., 63 (2013), 837-863. 1

[5] A. Aral, O. Doğru, Bleimann Butzer and Hahn operators based on q-integers, J. Inequal. Appl., 2007 (2007), 12 pages. 5

[6] S. Aytar, A characterization theorem for levelwise statistical convergence, Filomat, 25 (2011), 133-143. 5

[7] D. Bărbosu, Kantorovich-Stancu type operators, JIPAM. J. Inequal. Pure Appl. Math., 5 (2004), 6 pages. 1

[8] C. Bardaro, G. Vinti, P. L. Butzer, R. L. Stens, Kantorovich-type generalized sampling series in the setting of Orlicz spaces, Sampl. Theory Signal Image Process., 6 (2007), 29-52. 1

[9] D. Cárdenas-Morales, V. Gupta, Two families of Bernstein-Durrmeyer type operators, Appl. Math. Comput., 248 (2014), 342-353. 1

[10] X. Y. Chen, J. Q. Tan, Z. Liu, J. Xie, Approximation of functions by a new family of generalized Bernstein operators, J. Math. Anal. Appl., 450 (2017), 244-261. 1, 6

[11] J. de la Cal, A. M. Valle, A Generalization of Bernstein-Kantorovič Operators, J. Math. Anal. Appl., 252 (2000), $750-766$. 1

[12] N. Deo, M. Dhamija, D. Miclăuş, Stancu-Kantorovich operators based on inverse Pólya-Eggenberger distribution, Appl. Math. Comput., 273 (2016), 281-289. 1

[13] R. A. Devore, G. G. Lorentz, Constructive Approximation, Grundlehren der Mathematischen Wissenschaften, SpringerVerlag, New York-London, (1993). 2

[14] Z. Ditzian, V. Totik, Moduli of Smoothness, Springer-Verlag, New York, (1987). 3 
[15] O. Doğru, O. Duman, Statistical approximation by Meyer-König and Zeller operators based on q-integers, Publ. Math. Debrecen, 68 (2006), 199-214. 5

[16] O. Duman, C. Orhan, Statistical approximation by positive linear operators, Studia Math., 161 (2004), 187-197.

[17] E. Erkus, O. Duman, H. M. Srivastava, Statistical approximation of certain positive linear operators constructed by means of the Chan-Chyan-Srivastava polynomials, Appl. Math. Comput., 182 (2006), 213-222. 5

[18] A. D. Gadjiev, C. Orhan, Some approximation theorems via statistical convergence, Rocky Mountain J. Math., 32 (2002), 129-138. 5, 5.1

[19] H. Gonska, M. Heilmann, I. Raşa, Kantorovich operators of order k, Numer. Funct. Anal. Optim., 32 (2011), 717-738. 1

[20] H. Gonska, R. Pǎltănea, Simultaneous approximation by a class of Bernstein-Durrmeyer operators preserving linear functions, Czechoslovak Math. J., 60 (2010), 783-799. 1

[21] V. Gupta, A. M. Acu, D. F. Sofonea, Approximation of Baskakov type Pólya-Durrmeyer operators, Appl. Math. Comput., 294 (2017), 318-331. 1

[22] G. Içöz, A Kantorovich variant of a new type Bernstein-Stancu polynomials, Appl. Math. Comput., 218 (2012), $8552-$ 8560. 1

[23] A. Kajla, P. N. Agrawal, Szász-Durrmeyer type operators based on Charlier polynomials, Appl. Math. Comput., 268 (2015), 1001-1014. 5

[24] N. I. Mahamodov, Statistical approximation of Baskakov and Baskakov-Kantorovich operators based on q-integers, Cent. Eur. J. Math., 8 (2010), 816-826. 5

[25] M. A. Özarslan, H. Aktuğlu, Local approximation properties for certain King type operators, Filomat, 27 (2013), $173-181$. 2

[26] M. A. Özarslan, O. Duman, Smoothness properties of modified Bernstein-Kantorovich operators, Numer. Funct. Anal. Optim., 37 (2016), 92-105. 1

[27] Q. Razi, Approximation of a function by Kantorovich type operators, Mat. Vesnik, 41 (1989), 183-192. 1

[28] D. D. Stancu, The remainder in the approximation by a generalized Bernstein operator: a representation by a convex combination of second-order divided differences, Calcolo, 35 (1998), 53-62. 